\title{
Densely defined perturbed vector equilibrium problems
}

\author{
Tirth Ram and Parshotam Lal \\ Department of Mathematics University of Jammu, Jammu, India
}

Received: 8 August 2018, Accepted: 25 November 2018

Published online: 24 March 2019.

\begin{abstract}
In this work, we considered perturbed vector equilibrium problems involving set-valued monotone mapping and prove some existence results with and without compactness assumptions by employing KKM Fan lemma on self segment dense set, a special type of dense set, instead of whole domain.
\end{abstract}

Keywords: Perturbed vector equilibrium problems, self-segment-dense set, C-lower semicontinuity, monotone mapping and KKM map.

\section{Introduction}

Let $f: D \times D \rightarrow \mathbb{R}$ be a real function with $f(x, x)=0$ for all $x \in D$, where $D$ is a nonempty subset of $X$ and $X$ be a real topological vector space. Then the following problem, is to find $x \in D$ such that

$$
f(x, y) \geq 0, \text { for all } y \in D,
$$

is known as an classical single-valued equilibrium problem (In short, EP)[2].

This problem contains many problems as special cases for instance, optimization problem, problem of Nash equilibria, fixed point problems, variational inequalities and complementarity problems $[2,6]$ etc. Also, existence results for various type of equilibrium problems involving vector mapping in ordered vector space have been considered by many authors, see $[1,3,6,12]$. Recently, Laszlo and Viorel [8] obtaining existence results for the set-valued equilibrium problems formulated by Kristaly and Varga [7] by making the use of a special type of dense set i.e self-segment-dense set. Later on Salahuddin and Verma [11] use the same concept and obtaining existence results for generalized set-valued vector equilibrium problems. In this paper, motivated by the recent work $[8,11]$, we obtain the existence results for perturbed vector equilibrium problem formulated by $\mathrm{Fu}$ [4] and perturbed scalar equilibrium problem given by Blum and Oettli [2] both with and without compactness assumptions by using the concept of self-segment-dense set.

The concept of self-segment-dense set was introduced by Laszlo and Viorel [9] which is not same as that of a segment dense set introduced by Luc [10]. Although, in one dimensional case, the concepts of a segment-dense set and a self-segment-dense set are same and equivalent to the concept of a dense set. But in higher dimensions i.e greater than one, self-segment-dense subset possess certain special properties, characterized by Lemma 6, which play an important role in obtaining our existence results.

In an infinite dimensional real Hilbert space it is known that the unit sphere is dense in the unit ball with respect to the weak topology, but it is not self-segment-dense. This is a typical example of a dense set that is not self-segment-dense. 
We organize this paper as following. In the next section, we first introduced the necessary apparatus that we need and perturbed equilibrium problem and we also define the notion of a self-segment-dense set. Section 3 and 4 contain the main results of this paper, namely existence results for perturbed equilibrium problem and scalar equilibrium problems by making the use of self-segment-dense set.

\section{Preliminaries}

Let $Y$ be real locally convex Hausdorff space, and $C \subset Y$ a pointed, closed convex cone with apex at origin and with $\operatorname{int} C \neq \phi$. We say that $C$ satisfies the condition $(\Delta)$ if there is a pointed, closed convex cone $\widetilde{C}$ such that $C \backslash\{0\} \subset \widetilde{C}$. It is well-known that if $C$ has a base, then $C$ satisfies condition $(\Delta)$

Definition 1. [10] Let $X$ and $Y$ be real locally convex topological vector spaces, $C \subset Y$ a closed convex cone with apex at the origin, and $D \subset X$ a nonempty subset. Let $F: D \rightarrow 2^{Y}$ be a set-valued mapping. Then $F$ is said to be lower semicontinuous with respect to $C$ at $x \in D$ (in short, $C$-l.s.c ) if for any $y \in F(x)$ and each neighborhood $V$ of $Y, y$ there is a neighborhood $U$ of $x$ such that for each $z \in U \cap D, F(z) \cap(V+C) \neq \phi$ and $F$ is said to be $C$-l.s.c on $D$ if it is $C$-l.s.c at each $x \in D$.

Remark. (i) If $F$ is l.s.c at $x \in D$, then it is $C$-1.s.c at $x \in D$. (ii) If $F$ is single valued and $F$ is $(-C)$-l.s.c at $x \in D$, then it is $C$ - u.s.c at $x \in D$ in the sense of Tan and Tinh [13].

Definition 2. For a function $f: X \rightarrow \overline{\mathbb{R}}$ we denote by domf its domain, that is domf $=\{x \in X: f(x) \in \mathbb{R}\}$. We say that $f$ is upper semicontinuous at $x_{0} \in$ domf iff, for every $\varepsilon>0$, there exists a neighborhood $U$ of $x_{0}$ such that $f(x) \leq f\left(x_{0}\right)+\varepsilon$ for all $x \in U$. The function $f$ is called upper semicontinuous iff it is upper semicontinuous at every point of its domain.

Furthermore, we say that $f$ is lower semicontinuous at $x_{0} \in$ dom $f$ iff, for every $\varepsilon>0$, there exists a neighborhood $U$ of $x_{0}$ such that $f(x) \geq f\left(x_{0}\right)-\varepsilon$ for all $x \in U$. The function $f$ is called lower semicontinuous iff it is lower semicontinuous at every point of its domain.

Definition 3. Let $f: X \rightarrow 2^{\overline{\mathbb{R}}}$ be a function. Then

(i) $f$ is said to be upper semicontinuous at $x_{0}$ if and only if

$$
\lim _{x_{\alpha} \rightarrow x_{0}} \operatorname{supf}\left(x_{\alpha}\right) \leq f\left(x_{0}\right)
$$

where $\left\{x_{\alpha}\right\}$ is a net converging to $x_{0}$.

(ii) $f$ is said to be lower semicontinuous at $x_{0}$ if and only if

$$
\lim _{x_{\alpha} \rightarrow x_{0}} \inf f\left(x_{\alpha}\right) \geq f\left(x_{0}\right)
$$

where $\left\{x_{\alpha}\right\}$ is a net converging to $x_{0}$.

Definition 4. Let $X$ and $Y$ be topological vector spaces, $D \subset X$ a convex subset and $C \subset Y$ a convex cone. Let $F: D \rightarrow 2^{Y}$ and $G: D \times D \rightarrow 2^{Y}$ be given, then.

(i) $F$ is said to be $C$-convex if for any $x, y \in D, t \in[0,1]$, we have

$$
t F(x)+(1-t) F(y) \subset F(t x+(1-t) y)+C .
$$

(ii) $F$ is said to be $C$-concave if for any $x, y \in D, t \in[0,1]$, we have

$$
F(t x+(1-t) y) \subset t F(x)+(1-t) F(y)+C .
$$


(iii) $G$ is said to be monotone if for each $x, y \in D, G(x, y)+G(y, x) \subset-C$.

Lemma 1. [4] Let $D, Y$ and $C$ be as in definition 1 and $F: D \rightarrow 2^{Y}$ be C-l.s.c on D. Then the set $A=\{x \in D: F(x) \subset Y \backslash$ int $C\}$ is closed in $D$.

Lemma 2. [4] Let $D, Y$ and $C$ be as in Lemma 1 , and $F: D \rightarrow 2^{Y}$ be given. For any fixed $x, y \in D$, let $g(t)=T(t y+(1-$ $t) x), t \in[0,1]$. Assume that $g(t)$ is $(-C)$-l.s.c at $t=0$ and $\forall t \in(0,1], g(t) \subset Y \backslash(-$ int $C)$. Then $g(0) \subset Y \backslash(-$ intC $)$.

Definition 5. Let $D$ be a nonempty convex subset of a vector space $X$. A set-valued mapping $F: D \rightarrow 2^{X}$ is called KKMmapping if for each finite subset $\left\{x_{1}, x_{2} \ldots x_{n}\right\} \subset D$, we have

$$
\operatorname{co}\left\{x_{1}, x_{2} \cdots x_{n}\right\} \subseteq \bigcup_{i=1}^{n} F\left(x_{i}\right)
$$

where $\operatorname{co}(E)$ is a convex hull of a set $E$.

Lemma 3. (Fan Lemma ) [5] Let $X$ be a Hausdorff topological vector space, and let $D$ be a non empty convex subset of $X$. Let $F: D \rightarrow 2^{X}$ be a KKM -mapping. If each $F(x)$ is closed and at least one $F(x)$ is compact, then $\bigcap_{x \in D} F(x) \neq \phi$.

Now we will work for the following equilibrium problems.

Let $X$ and $Y$ be real topological vector spaces, $D \subset X$ a nonempty subset and $C \subset Y$ a closed, pointed and convex cone with apex at origin and $\operatorname{int} C \neq \phi$. Let $G, H: D \times D \rightarrow 2^{Y}$ be set valued mappings. Then we consider the following Perturbed vector equilibrium problems:

(PVEP 1) Find $x_{0} \in D$ such that $G\left(x_{0}, y\right)+H\left(x_{0}, y\right) \subset Y \backslash(-i n t C), \forall y \in D$.

(PVEP 2) Find $x_{0} \in D$ such that $\left[G\left(x_{0}, y\right)+H\left(x_{0}, y\right)\right] \cap(Y \backslash(-i n t C)) \neq \phi, \forall y \in D$.

(PVEP 3) Find $x_{0} \in D$ such that $G\left(x_{0}, y\right)+H\left(x_{0}, y\right) \subset Y \backslash(-C \backslash\{0\}), \forall y \in D$.

If $G$ and $H$ are single valued mappings, then the problems (PVEP 1) and (PVEP 2) both collapse to the following VEP equilibrium problems:

(PVEP 4) Find $x_{0} \in D$ such that $G\left(x_{0}, y\right)+H\left(x_{0}, y\right) \in Y \backslash(-i n t C), \forall y \in D$.

(PVEP 5) Find $x_{0} \in D$ such that $G\left(x_{0}, y\right)+H\left(x_{0}, y\right) \in Y \backslash(-C \backslash\{0\}), \forall y \in D$.

The scalar equilibrium problem introduced and studied by Blum and Oettli [2], for sum of two bifunctions $f, g: K \times K \longrightarrow \mathbb{R}$ consists in finding $x_{0} \in K$ such that

$$
f\left(x_{0}, y\right)+g\left(x_{0}, y\right) \geq 0, \forall y \in K
$$

Now we shall recall the original existence results of $\mathrm{Fu}[10]$ regarding the set valued vector equilibrium problems (VEP1) and Blum and Oettli [2] for (EP).

Lemma 4. ([4], Lemma 4) Let $X$ and $Y$ be real locally convex Hausdorff topological spaces, $D$ a nonempty closed, convex subset of $X$ and $C \subset Y$ a pointed, closed convex cone with apex at origin and int $C \neq \phi$. Let $G, H: D \times D \rightarrow 2^{Y}$ be set-valued mappings satisfing the following conditions:

(i) For all $x \in D, 0 \in G(x, x) \subset C, 0 \in H(x, x) \subset C$,

(ii) $G$ is monotone,

(iii) For any fixed $x, y \in D$, the mapping $g(t):=G(t y+(1-t) x), t \in[0,1]$, is $(-C)$ - l.s.c at $t=0$,

(iv) For any fixed $x \in D, G(x,),. H(x,):. D \longrightarrow 2^{Y}$ are $C$-convex.

Then the following are equivalent: 
(I) There exists $x^{*} \in D, G\left(y, x^{*}\right)-H\left(x^{*}, y\right) \subset Y \backslash($ int $C), \forall y \in D$,

(II) There exists $x^{*} \in D, G\left(x^{*}, y\right)+H\left(x^{*}, y\right) \subset Y \backslash(-\operatorname{int} C), \forall y \in D$.

Theorem 1. ([4], Lemma 5) Let $K$ be a nonempty compact subset of $X$ and $G, H: K \times K \rightarrow 2^{Y}$ be a set-valued mappings such that:

(i) For all $x \in K, 0 \in G(x, x) \subset C, 0 \in H(x, x) \subset C$,

(ii) $G$ is monotone,

(iii) For any fixed $x, y \in K$, the mapping $g(t):=G(t y+(1-t) x), t \in[0,1]$, is $(-C)$-l.s.c at $t=0$,

(iv) For any fixed $x \in K, G(x,),. H(x,):. K \longrightarrow 2^{Y}$ are $C$-convex,

(v) For any fixed $x \in K, G(x, y)$ is $C$-l.s.c in $y$, and for any fixed $y \in K, H(x, y)$ is $(-C)$-l.s.c in $x$.

Then, there exists $x^{*} \in K$ such that

$$
G\left(x^{*}, y\right)+H\left(x^{*}, y\right) \subset Y \backslash(-i n t C), \forall y \in K \text {. }
$$

Theorem 2. ([4], Theorem 2) Let D, C, G and H be as in Lemma 4, and let all conditions (i)-(v) of Theorem 10 holds. In addition, $G$ and $H$ satisfy the following condition:

(vi)there exists a nonempty compact convex subset $K \subset D$ such that, $\forall x \in D \backslash K, \exists a \in K$,

$$
G(a, x)-H(x, a) \not \subset Y \backslash \operatorname{int} C
$$

Then, there exists $x^{*} \in K$ such that

$$
G\left(x^{*}, y\right)+H\left(x^{*}, y\right) \subset Y \backslash(-i n t C), \forall y \in D .
$$

If $C$ satisfies the condition $(\Delta)$, then

$$
G\left(x^{*}, y\right)+H\left(x^{*}, y\right) \subset Y \backslash(-C \backslash\{0\}), \forall y \in D .
$$

Lemma 5. [2] Let $X$ be a real topological vector spaces and $K \subset X$ be a compact convex nonempty set. Let $g, h: K \times K \rightarrow$ $\mathbb{R}$ be two functions satisfies following:

(i) For all $x \in K, g(x, x)=0, h(x, x)=0$,

(ii) $g$ is monotone,

(iii) For all $x, y \in K$, the mapping $t \in[0,1] \mapsto g(t y+(1-t) x, y)$, is u.s.c at $t=0$, (hemicontinuity)

(iv) For all $x \in K, g(x,),. h(x,):. K \longrightarrow \mathbb{R}$, are convex.

Then the fillowing are equivalent:

(I) There exists $x_{0} \in K, g\left(y, x_{0}\right)-h\left(x_{0}, y\right) \leq 0, \forall y \in K$,

(II)There exists $x_{0} \in K, g\left(x_{0}, y\right)+h\left(x_{0}, y\right) \geq 0, \forall y \in K$,

Theorem 3. [2] Let $X$ be a real topological vector spaces and $K \subset X$ be a compact convex nonempty set. Let $g$, $h$ : $K \times K \rightarrow \mathbb{R}$ be two functions satisfies following:

(i) For all $x \in K, g(x, x)=0, h(x, x)=0$,

(ii) $g$ is monotone,

(iii) For all $x, y \in K$, the mapping $t \in[0,1] \mapsto g(t y+(1-t) x, y)$, is u.s.c at $t=0 \quad$ (hemicontinuity),

(iv) For all $x \in K, g(x,),. h(x,):. K \longrightarrow \mathbb{R}$, are convex,

(v) For all $x \in K, g(x,$.$) is l.s.c and for all y \in K, h(., y)$, is u.s.c.

Then, there exists $x_{0} \in K$ such that

$$
g\left(x_{0}, y\right)+h\left(x_{0}, y\right) \geq 0, \forall y \in K
$$


Theorem 4. [2] Let $X$ be a real topological vector spaces and $K \subset X$ be a closed, convex nonempty set. Let $g, h: K \times K \rightarrow$ $\mathbb{R}$ be two functions satisfies following:

(i) For all $x \in K, g(x, x)=0, h(x, x)=0$,

(ii) $g$ is monotone,

(iii) For all $x, y \in K$, the mapping $t \in[0,1] \mapsto g(t y+(1-t) x, y)$, is u.s.c at $t=0$,

(iv) For all $x \in K, g(x,),. h(x,):. K \longrightarrow \mathbb{R}$, are convex,

(v) For all $x \in K, g(x,$.$) is l.s.c and for all y \in K, h(., y)$, is u.s.c,

(vi) There exists a nonempty compact convex set $B \subset K$ such that for any $x \in K \backslash B$, there exists $a \in B$ with

$$
g(x, a)+h(x, a)<0 .
$$

Then, there exists $x_{0} \in B$ such that

$$
g\left(x_{0}, y\right)+h\left(x_{0}, y\right) \geq 0, \quad \forall y \in K
$$

Self-segment dense set. Let $X$ be a Hausdorff topological vector space. We will use following notations for the open, respectively closed, line segments in $X$ with the endpoints $\mathrm{x}$ and $\mathrm{y}$

$$
\begin{aligned}
& (x, y)=\{z \in X: z=x+t(y-x), t \in(0,1)\}, \\
& {[0,1]=\{z \in X: z=x+t(y-x), t \in[0,1]\} .}
\end{aligned}
$$

In [10], Definition 4, The Luc has introduced the notion of a so-called segment-dense set. Let $V \subseteq X$ be a convex set. One says that the set $U \subseteq V$ is segment-dense in $V$ if for each $x \in V$ there can be found $y \in U$ such that $x$ is a cluster point of the set $[x, y] \cap U$.

Laszlo and Viorel [9] presented a denseness notion which is slightly different from the concept of the Luc [10] presented above.

Consider the sets $U \subseteq V \subseteq X$ and assume that $V$ is convex. We say that $U$ is self-segment-dense in $V$ if $U$ is dense in $V$ and

$$
\forall x, y \in U, \text { the set }[x, y] \cap U \text { is dense in }[x, y] .
$$

\section{Self-segment-dense set and vector equilibrium problems}

Lemma 6. ([8], Lemma 1) Let $X$ be a Hausdroff locally convex topological vector space, let $V \subseteq X$ be a convex set and let $U \subseteq V$ a self-segment-dense in $V$. Than for all finite subset $\left\{u_{1}, u_{2} \cdots u_{n}\right\} \subseteq U$ one has

$$
\operatorname{cl}\left(\operatorname{co}\left\{u_{1}, u_{2} \cdots u_{n}\right\} \cap D\right)=\operatorname{co}\left\{u_{1}, u_{2} \cdots u_{n}\right\}
$$

Theorem 5. Let $X$ be a Hausdroff locally convex topological vector space, and let $K$ be a nonempty convex, compact subset of $X$, let $D \subseteq K$ be a self-segment-dense, and let $G, H: K \times K \rightarrow 2^{Y}$ be set-valued mappings satisfying:

(i) For all $x \in D, 0 \in G(x, x) \subset C, 0 \in H(x, x) \subset C$,

(ii) $G$ is monotone,

(iii) For any fixed $x, y \in D$, the mapping $g(t)=G(t y+(1-t) x, y), t \in[0,1]$, is $(-C)-$ l.s.c at $t=0$,

(iv) For any fixed $x \in D, G(x,),. H(x,):. D \rightarrow 2^{Y}$ are $C$-convex,

(v) For any fixed $x \in D, G(x, y)$ is $C$-l.s.c in $y$ on $K$ and for any fixed $y \in D, H(x, y)$ is $(-C)$-l.s.c in $x$ on $K$,

(vi) For any fixed $y \in K, G(x, y)$ is $C$-l.s.c in $x$ on $K \backslash D$, for any fixed $x \in K, H(x, y)$ is $(-C)$-l.s.c in $y$ on $K \backslash D$. 
Then, there exists $x^{*} \in K$ such that

$$
G\left(x^{*}, y\right)+H\left(x^{*}, y\right) \subset Y \backslash(-i n t C), \forall y \in K .
$$

Proof. Define $F: D \rightarrow 2^{K}$ by

$$
F(y)=\{x \in K: G(y, x)-H(x, y) \subset Y \backslash(\text { int } C)\}, \forall y \in D
$$

Since for fixed $y \in D, G(y,)-.H(., y)$ is $C-$ l.s.c on $D$, so $F(y)$ is closed in $K$ and hence compact.

Claim: $F$ is a $K K M$ mapping. i.e For any $\left\{y_{1}, y_{2} \cdots y_{n}\right\} \subset D$, we have

$$
\operatorname{co}\left\{y_{1}, y_{2} \cdots y_{n}\right\} \cap D \subseteq \bigcup_{i=1}^{n} F\left(y_{i}\right) .
$$

Suppose not, then there exists $\left\{y_{1}, y_{2} \cdots y_{n}\right\} \subset D$ and $t_{1}, t_{2} \cdots t_{n}$ with $\sum_{i=1}^{n} t_{i}=1$ and $z=\sum_{i=1}^{n} t_{i} y_{i} \in D$ such that $z \notin \bigcup_{i=1}^{n} F\left(y_{i}\right)$

$$
\begin{aligned}
& \Rightarrow z \notin F\left(y_{i}\right), \forall i=1,2, \cdots, n . \\
& \Rightarrow G\left(y_{i}, z\right)-H\left(z, y_{i}\right) \not \subset Y \backslash \operatorname{int} C, \forall i=1, . ., n . \\
& \Rightarrow G\left(y_{i}, z\right)-H\left(z, y_{i}\right) \subset \operatorname{int} C, \forall i=1, . ., n .
\end{aligned}
$$

This implies that there exists $a_{i} \in G\left(y_{i}, z\right), b_{i} \in H\left(z, y_{i}\right)$ such that $a_{i}-b_{i} \in \operatorname{int} C, \forall i=1, . ., n$.

$$
\Rightarrow \sum_{i}^{n} t_{i}\left(a_{i}-b_{i}\right) \in \operatorname{int} C
$$

Since $G$ is monotone and $C$-convex in second argument, it follows that

$$
\sum_{i=1}^{n} t_{i} G\left(y_{i}, z\right) \subset-C-\sum_{i=1}^{n} t_{i} G\left(z, y_{i}\right) \subset-C-G(z, z)-C \subset-C .
$$

Also $H$ is $C$-convex in second argument

$$
\sum_{i=1}^{n} t_{i} H\left(z, y_{i}\right) \subset H(z, z)+C \subset C+C=C .
$$

From (2) and (3), we get

$$
\sum_{i=1}^{n} t_{i}\left(G\left(y_{i}, z\right)-H(z, y i)\right) \subset-C
$$

From (1) and (4), we have

$$
\sum_{i=1}^{n}\left(G\left(y_{i}, z\right)-H\left(z, y_{i}\right)\right) \subset(-C) \cap i n t C=\phi,
$$


a contradiction. Hence, $c o\left\{y_{1}, y_{2} \cdots y_{n}\right\} \cap D \subseteq \bigcup_{i=1}^{n} F\left(y_{i}\right)$.

$$
\Rightarrow \operatorname{cl}\left(\operatorname{co}\left\{y_{1}, y_{2} \cdots y_{n}\right\} \cap D\right) \subseteq \operatorname{cl}\left(\bigcup_{i=1}^{n} F\left(y_{i}\right)\right)
$$

By Lemma 6, $\operatorname{cl}\left(\operatorname{co}\left\{y_{1}, y_{2} \cdots, y_{n}\right\} \cap D\right)=\operatorname{co}\left\{y_{1}, y_{2} \cdots y_{n}\right\}$ and also we know that $\operatorname{cl}\left(\bigcup_{i=1}^{n} F\left(y_{i}\right)\right)=\left(\bigcup_{i=1}^{n} F\left(y_{i}\right)\right)$ Hence from (5), we have

$$
\operatorname{co}\left\{y_{1}, y_{2} \cdots y_{n}\right\} \subseteq \bigcup_{i=1}^{n} F\left(y_{i}\right)
$$

Hence $F$ is a KKM- mapping, So by Ky-Fan lemma,

$$
\bigcap_{y \in D} F(y) \neq \phi
$$

i.e. there exists $x^{*} \in K$ such that

$$
G\left(y, x^{*}\right)-H\left(x^{*}, y\right) \subset y \backslash(\operatorname{int} C), \forall y \in D .
$$

To complete the proof we have to show that,

$$
\bigcap_{y \in K} F(y) \neq \phi
$$

Suppose there exists $y^{*} \in K \backslash D$ such that

$$
\begin{aligned}
& G\left(y^{*}, x\right)-H\left(x, y^{*}\right) \not \subset Y \backslash \operatorname{int} C . \\
& \Rightarrow G\left(y^{*}, x\right)-H\left(x, y^{*}\right) \subset \operatorname{int} C .
\end{aligned}
$$

Since int $C$ is a neighborhood of $F\left(y^{*}\right)$, where $F\left(y^{*}\right)=G\left(y^{*}, x\right)-H\left(x, y^{*}\right)$, and $F$ is $C$-l.s.c on $K \backslash D$, there exists a neighborhood $U$ of $y^{*}$ such that

$$
\begin{aligned}
& F(y) \cap(\operatorname{int} C+C) \neq \phi, \forall y \in U . \\
& \Rightarrow F(y) \cap(\operatorname{int} C) \neq \phi, \forall y \in U .
\end{aligned}
$$

Since $D$ is dense in $K$, so there exists $y_{1} \in U$ and $y_{1} \in D$ such that

$$
F\left(y_{1}\right) \cap \operatorname{int} C \neq \phi
$$

a contradiction to (6). Hence, $\bigcap_{y \in K} F(y) \neq \phi$, i.e. there exists $x^{*} \in K$ such that

$$
G\left(y, x^{*}\right)-H\left(x^{*}, y\right) \subset Y \backslash(\text { int } C), \forall y \in K
$$

Hence by Lemma 4, there exists $x^{*} \in K$ such that

$$
G\left(x^{*}, y\right)+H\left(x^{*}, y\right) \subset Y \backslash(-i n t C), \forall y \in K
$$

Theorem 6. Let $D \subseteq K$ be a self-segment dense set and $K$ is a compact subset of $X$. Let $g, h: K \times K \rightarrow \mathbb{R}$ be two single valued functions satisfying following conditions:

(i) For all $x \in D, g(x, x)=0, h(x, x)=0$, 
(ii) $g$ is monotone,

(iii) For all $x, y \in D$, the mapping $t \in[0,1] \mapsto g(t y+(1-t) x, y)$, is u.s.c at $t=0$.

(iv) For all $x \in D, g(x,),. h(x,):. K \longrightarrow \mathbb{R}$, are convex,

(v) For all $x \in D, g(x,$.$) is l.s.c on K$ and for all $y \in D, h(., y)$, is u.s.c on $K$,

(vi) For all $y \in K, g(., y)$, is l.s.c on $K \backslash D$ and for all $x \in K, h(x,$.$) is u.s.c on K \backslash D$.

Then, there exists $x_{0} \in K$ such that

$$
g\left(x_{0}, y\right)+h\left(x_{0}, y\right) \geq 0, \forall y \in K
$$

Proof. Define a set-valued map $F: D \longrightarrow 2^{K}$ by

$$
F(y):=\{x \in K: g(y, x)-h(x, y) \leq 0\}, \forall y \in D
$$

Since for fixed $y \in D, g(y,)-.h(., y)$ l.s.c on $D, F(y)$ is closed in $K$ and $K$ is compact and hence $F(y)$ is compact, $\forall y \in D$.

Claim: $F$ is a $K K M-m a p$. i.e. For any finite set $\left\{y_{1}, y_{2}, \cdots y_{n}\right\} \subset D$, we have

$$
\operatorname{co}\left\{y_{1}, y_{2}, \ldots, y_{n}\right\} \bigcap D \subseteq \bigcup_{i=1}^{n} F\left(y_{i}\right)
$$

Suppose not, then there exists $\left\{y_{1}, y_{2}, \cdots y_{n}\right\} \subset D$ and $t_{1}, t_{2}, . ., t_{n}$ with $\sum_{i=1}^{n} t_{i}=1$ and $z=\sum_{i=1}^{n} t_{i} y_{i} \in D$ such that $z \notin \bigcup_{i=1}^{n} F\left(y_{i}\right)$

$$
\begin{aligned}
& \Rightarrow z \notin F\left(y_{i}\right), \forall i=1,2, \cdots n . \\
& \Rightarrow g\left(y_{i}, z\right)-h\left(z, y_{i}\right)>0, \forall i=1,2, \cdots n . \\
& \Rightarrow g\left(y_{i}, z\right)>h\left(z, y_{i}\right), \forall i=1,2, \cdots n . \\
& \Rightarrow \sum_{i=1}^{n} \mu_{i} g\left(y_{i}, z\right)>\sum_{i=1}^{n} \mu_{i} h\left(z, y_{i}\right) .
\end{aligned}
$$

Since $g$ is monotone and convex in second argument, we have

$$
\sum_{i=1}^{n} \mu_{i} g\left(y_{i}, z\right) \leq \sum_{i=1}^{n} \sum_{j=1}^{n} \mu_{i} \mu_{j} g\left(y_{j}, y_{i}\right) \leq \frac{1}{2} \sum_{i=1}^{n} \mu_{i} \mu_{j}\left(g\left(y_{i}, y_{j}\right)+g\left(y_{j}, y_{i}\right)\right) \leq 0
$$

Since $h$ is $C$-convex in second argument

$$
0=h(z, z) \leq \sum_{i=1}^{n} \mu_{i} h\left(z, y_{i}\right)
$$

From (8) and (9), we have

$$
\sum_{i=1}^{n} \mu_{i} g\left(y_{i}, z\right) \leq 0 \leq \sum_{i=1}^{n} \mu_{i} h\left(z, y_{i}\right)
$$

a contradiction to (7). Hence, $\operatorname{co}\left(\left\{y_{1}, y_{2}, \ldots, y_{n}\right\}\right) \bigcap D \subseteq \bigcup_{i=1}^{n} F\left(y_{i}\right)$

$$
\Rightarrow \operatorname{cl}\left(\operatorname{co}\left(\left\{y_{1}, y_{2}, \cdots, y_{n}\right\}\right) \bigcap D\right) \subseteq \operatorname{cl}\left(\bigcup_{i=1}^{n} F\left(y_{i}\right)\right)
$$


By Lemma $6, \operatorname{cl}\left(\operatorname{co}\left(\left\{y_{1}, y_{2}, \cdots n\right\}\right) \cap D\right)=\operatorname{co}\left(\left\{y_{1}, y_{2}, \cdots y_{n}\right\}\right)$ and we also know that

$$
\operatorname{cl}\left(\bigcup_{i=1}^{n} F\left(y_{i}\right)\right)=\bigcup_{i=1}^{n} F\left(y_{i}\right)
$$

Thus, from (10), we have

$$
\operatorname{co}\left(\left\{y_{1}, y_{2}, \cdots, y_{n}\right\}\right) \subseteq \bigcup_{i=1}^{n} F\left(y_{i}\right) .
$$

Hence, $F$ is a $K K M-$ map. So, by Fan Lemma, we have

$$
\bigcap_{y \in D} F(y) \neq \phi
$$

i.e., there exists $x_{0} \in K$ such that

$$
g\left(y, x_{0}\right)-h\left(x_{0}, y\right) \leq 0, \forall y \in D .
$$

To complete the poof we need to show that,

$$
\bigcap_{y \in K} F(y) \neq \phi
$$

i.e., there exists $x_{0} \in K$ such that

$$
g\left(y, x_{0}\right)-h\left(x_{0}, y\right) \leq 0, \forall y \in K .
$$

Suppose there exists $y \in K \backslash D$, Since $D$ is dense in $K$, there exists a net $\left\{y_{\alpha}\right\} \subset D$ such that $y_{\alpha} \longrightarrow y$. Since $K()=.g\left(., x_{0}\right)-h\left(x_{0},.\right)$ is 1.s.c in $K \backslash D$ and $y_{\alpha} \longrightarrow y$, so

$$
0 \geq \lim _{y_{\alpha} \longrightarrow y} \inf K\left(y_{\alpha}\right) \geq K(y)=g\left(y, x_{0}\right)-h\left(x_{0}, y\right)
$$

Hence,

$$
\bigcap_{y \in K} F(y) \neq \phi .
$$

i.e., there exists $x_{0} \in K$ such that

$$
g\left(y, x_{0}\right)-h\left(x_{0}, y\right) \leq 0, \forall y \in K .
$$

Hence by Lemma 5 , there exists $x_{0} \in K$ such that

$$
g\left(x_{0}, y\right)+h\left(x_{0}, y\right) \geq 0, \forall y \in K
$$

This complete the proof.

\section{Set-valued vector equilibrium problems without compactness assumptions}

Theorem 7. Let $K$ be a nonempty convex closed subset of $X$, let $D \subseteq K$ be a self-segment-dense, and let $G, H: K \times K \rightarrow 2^{Y}$ be set-valued mappings satisfying:

(i) For all $x \in D, 0 \in G(x, x) \subset C, 0 \in H(x, x) \subset C$,

(ii) $G$ is monotone,

(iii) For any fixed $x, y \in D$, the mapping $g(t):=G(t y+(1-t) x, y), t \in[0,1]$, is $(-C)-$ l.s.c at $t=0$,

(iv) For any fixed $x \in D, G(x,),. H(x,):. D \rightarrow 2^{Y}$ are $C$-convex,

(v) For any fixed $x \in D, G(x, y)$ is $C$-l.s.c in $y$ on $K$ and for any fixed $y \in D, H(x, y)$ is $(-C)$-l.s.c in $x$ on $K$,

(vi) For any fixed $y \in K, G(x, y)$ is $C$-l.s.c in $x$ on $K \backslash D$ and for any fixed $x \in K, H(x, y)$ is $(-C)$-l.s.c in $y$ on $K \backslash D$, 
(vii) There exists a compact set $K_{0} \subseteq D$ and $y_{0} \in D \cap K_{0}$. such that $G\left(y_{0}, x\right)-H\left(x, y_{0}\right) \not \subset Y \backslash$ int $C, \forall x \in K \backslash K_{0}$. Then, there exists $x^{*} \in K_{0}$ such that

$$
G\left(x^{*}, y\right)+H\left(x^{*}, y\right) \subset Y \backslash(-i n t C), \forall y \in K \text {. }
$$

Proof. Define a set-valued map $F: D \rightarrow 2^{K}$ by

$$
F(y)=\{x \in K: G(y, x)-H(x, y) \subset Y \backslash(\text { int } C)\}, \forall y \in D .
$$

According to the proof of Theorem $5, F(y)$ is closed in $K$, for all $y \in D$.

Next we shall show that $F\left(y_{0}\right)$ is compact, and the rest of the proof is similar to the proof of Theorem 5. For this it is enough to show that $F\left(y_{0}\right) \subseteq K_{0}$. Suppose $F\left(y_{0}\right) \nsubseteq K_{0}$, then there exists $z \in F\left(y_{0}\right)$ such that $z \notin K_{0}$.

Now, $z \in F\left(y_{0}\right)$, so $G\left(y_{0}, z\right)-H\left(z, y_{0}\right) \subset Y \backslash \operatorname{int} C$, which is a contradiction to (vii).

Theorem 8. Let $D \subseteq K$ be a self-segment dense set and $K$ is nonempty closed convex subset of $X$. Let $g, h: K \times K \rightarrow \mathbb{R}$ be two single valued functions satisfying following conditions:

(i) For all $x \in D, g(x, x)=0, h(x, x)=0$,

(ii) $g$ is monotone,

(iii) For all $x, y \in D$, the mapping $t \in[0,1] \longrightarrow g(t y+(1-t) x, y)$, is u.s.c at $t=0$,

(iv) For all $x \in D, g(x,),. h(x,):. K \longrightarrow \mathbb{R}$ are convex,

(v) For all $x \in D, g(x,$.$) , is l.s.c on K$ and for all $y \in D, h(., y)$ is u.s.c on $K$,

(vi) For all $y \in K, g(., y)$ is l.s.c on $K \backslash D$ and for all $x \in D, h(x,$.$) is u.s.c on K \backslash D$,

(vii) There exists a compact set $K_{0} \subseteq D$ such that for all $y \in D \cap K_{0}$, s.t. $g\left(y_{0}, x\right)-h\left(x, y_{0}\right)>0$, for all $x \in K \backslash K_{0}$,

Then, there exists $x_{0} \in K_{0}$ such that

$$
g\left(x_{0}, y\right)+h\left(x_{0}, y\right) \geq 0, \forall y \in K
$$

Proof. Define a set-valued map $F: D \rightarrow 2^{K}$

$$
F(y)=\{x \in K: g(y, x)-h(x, y) \leq 0\}, \forall y \in D
$$

According to the proof of Theorem $6, F(y)$ is closed in $K, \forall y \in D$.

Next we shall show that $F\left(y_{0}\right)$ is compact, and then the rest of the proof is similar to the proof of Theorem 6 , For this it is enough to show that $F\left(y_{0}\right) \subseteq K_{0}$. Suppose $F\left(y_{0}\right) \nsubseteq K_{0}$, then $\exists z \in F\left(y_{0}\right)$ such that $z \notin K_{0}$.

Now, $z \in F\left(y_{0}\right)$, so $g\left(y_{0}, z\right)-h\left(z, y_{0}\right) \leq 0$, which is a contradiction to (vii).

\section{Competing interests}

The authors declare that they have no competing interests.

\section{Authors' contributions}

All authors have contributed to all parts of the article. All authors read and approved the final manuscript. 


\section{References}

[1] Q.H. Ansari, W. Oettle and D. Schlager, A generalization of vector equilibria, Mathematical Methods of Operational Research 46 (1997), 147-152.

[2] E. Blum and W. Oettli, From optimization and variational inequalities to equilibrium problems,Mathematics Student, 63 (1994), 123-145.

[3] J.Y. Fu, Generalized vector quasi-equilibrium problems. Mathematical Methods of Operational Research 52(2000), 57-64.

[4] J.Y. Fu, Vector equilibrium problems. Existence theorems and convexity of solution set, Journal of Global Optimization 31(2005), 109-119.

[5] K. Fan, A minimax inequality and applications, in Inequalities III, Shisha, Academic Press, (1972), 103-113.

[6] F. Giannessi, Vector variational inequality and vector equilibria: Mathematical Theories, Kluwer Academic Publishers, Dordrecht/ Boston/ London, 2000.

[7] A. Kristaly and C. Varga, Set-valued version of Ky-Fan's inequality with applications to variational inclusions theory,Journal of Mathematical Analysis and Applications 282 (2003), 8-20.

[8] S. Laszlo and A. Viorel, Densely defined equilibrium problems, Journal of optimization theory and applications 21 (2015).

[9] S.C. Laszlo and A. Viorel, Generalized monotone operators on dense sets, Numerical Functional Analysis and Optimization P36(2015),901-929.

[10] D.T. Luc, Existence results for densely defined pseudomonotone variational inequalities, Journal of Mathematical analysis and applications 254(2001), 291-308.

[11] Salahuddin and R.U. Verma, Generalized set-valued vector equilibrium problems, PanAmerican Mathematical Journal 27(2017), 79-97.

[12] W.Song, Vector equilibrium problems with set-valued mappings. Appears in Vector variational inequality and vector equilibria: Mathematical Theories, Kluwer Academic Publishers, Dordrecht/ Boston/ London,2000, 403-422.

[13] N.X. Tan and P.N. Tinh, On the existence of equilibrium points of vector functions, Numer.Funct. Anal. Optim. 19(1-2)(1998), 141-156. 